\title{
Funcionamiento familiar y su relación con la interacción social en niños con el Trastorno del
}

\section{Espectro Autista}

Family functioning and its relation to social interaction in children with the disorder of The Autistic Spectrum

Funcionamiento familiar - interacción social

Inger Maitta Rosado(1)

María Lazo Moreira(2)

Enita Sánchez Bravo(3)

(1) Universidad Técnica de Manabí. Portoviejo. Ecuador. e-mail: ingermaitta7@gmail.com

(2) Universidad Técnica de Manabí. Portoviejo. Ecuador. e-mail: psicvlazom@ @otmail.com

(3) Egresada de Psicología Clínica. e-mail: eny_1725@ @otmail.com

Contacto: eny_1725@hotmail.com

\section{Recibido: 7-9-2020 Aprobado: 19-12-2020}

\section{Resumen}

El objetivo del estudio fue evaluar la interacción social de niños y niñas con el Trastorno del Espectro Autista, la "Fundación un lugar para tus sueños" de la ciudad de Portoviejo-Manabí, Ecuador. El enfoque de esta investigación es cualitativo, a través del método deductivo inductivo establece una relación entre ambas variables, apoyado en el método bibliográfico y estadístico, se aplicó el instrumento del APGAR familiar y un cuestionario para evaluar la interacción social de los/las niños/as participantes. Como resultado se obtuvo que la mayor parte de las familias tienen una función familiar normal y la interacción social de sus hijos oscila entre Buena y Regular, indicando que los niveles de funcionalidad familiar y la interacción social de los/las niños/as con TEA está íntimamente relacionado, pues el rol que cumplen los miembros de la familia es indispensables para la adaptación social del niño/a.

Palabras clave: Familia, interacción social, espectro autista, funcionalidad familiar.

\section{Abstract}


The objective of the study was to evaluate the social interaction of boys and girls with Autism Spectrum Disorder, the "Foundation a place for your dreams" of the city of Portoviejo-Manabí, Ecuador. The focus of this research is qualitative, through the inductive deductive method it establishes a relationship between both variables, supported by the bibliographic and statistical method, the family APGAR instrument and a questionnaire were applied to evaluate the social interaction of the children / the participants. As a result, it was obtained that most of the families have a normal family function and the social interaction of their children oscillates between Good and Fair, indicating that the levels of family functionality and the social interaction of children with ASD are closely related, since the role played by family members is essential for the child's social adaptation.

Keywords: Family, social interaction; Autistic Spectrum; family functionality.

\section{Introducción}

Según Montagut, D'Ocon, \& Pastor (2017) el término trastorno del espectro autista (TEA) (ASD, por sus siglas en inglés, Autism Spectrum Disorders), hace referencia a un conjunto de trastornos del neurodesarrollo que se distinguen de otros trastornos por presentar alteraciones de origen cognitivo, conductual y en áreas de la comunicación principalmente. El trastorno del espectro autista, antes llamado simplemente como autismo, está caracterizado por alteraciones en la comunicación, la interacción social y un comportamiento particular que tiene el paciente, este trastorno se lo diagnostica en etapas tempranas del desarrollo, generalmente a partir de los 18 meses de edad (Oviedo, Apolinar, de la Chesnaye, \& Guerra, 2015).

La gravedad de estas manifestaciones marcan el grado del trastorno, que se clasifica en tres, considerando el primero como el nivel de menor afectación y el tercero de mayor afectación siendo este último el de mayor necesidad de apoyo (Asociación Americana de Psiquiatría, 2013). De acuerdo a American Psychiatric Association (2000) el TEA comprende trastornos que en la actualidad se conocen como el Síndrome de Asperger, Síndrome de Rett, y Trastorno Desintegrativo Infantil.

El trastorno del espectro autista (TEA) se refiere a un conjunto de discapacidades del desarrollo que pueden causar impactos sociales, de comunicación y problemas del comportamiento. El autismo es considerado un desorden conocido con el término espectro, lo que significa que todo aquello que lo caracteriza, como sus síntomas por ejemplo, pueden variar, es decir, tiene una extensa diversidad de combinaciones en las que puede presentarse (Miguel, 2006; Baña, 2015; Coy \& Martín, 2017). 
Morales, (2010), define al autismo como una entidad psicológica diferenciada por un conjunto de rasgos (denominado triada autista) que siguen un curso temporal característicos a partir de los 18 meses de vida, donde el propio proceso de construcción de la experiencia se ve afectado. El funcionamiento familiar y su relación con la interacción social en niños con Trastorno del Espectro Autista es un tema de suma importancia conocer, debido a que la interacción social y la comunicación son las áreas más afectadas de este trastorno, por lo que desenvolverse eficazmente en el entorno social, es un gran reto para los padres y familiares de este sujeto.

En este sentido se ha notificado que afecta a las personas de diferentes formas y puede variar de leve a grave. Las personas con autismo comparten algunos síntomas, como las dificultades con la interacción social pero existen diferencias en los síntomas, porque cada persona es diferente y el grado de afectación también puede variar, además de que pueden existir otros tipos de problemas.

El presente artículo tiene por objetivo, evaluar la interacción social de niños y niñas con el Trastorno del Espectro Autista, la "Fundación un lugar para tus sueños" de la ciudad de PortoviejoManabí, Ecuador.

\section{Conceptualización de familia}

Para referirse a la palabra familia, se habla de una unidad básica de la sociedad, en la que la existencia de cada miembro hace posible la totalidad de dicho sistema, en él se comparten fortalezas, debilidades, y aprendizajes propios y de la realidad su entorno (Zavala, Ríos, García, \& Rodríguez, 2009).

Mendoza et al. (2006), hace alusión a la familia y la denomina como un sistema que a su vez está compuesto por subsistemas, y que en dicha composición tenemos al subsistema conyugal (papá y mamá), subsistema paterno-filial (padres e hijos) y subsistema fraternal (hermanos), toda familia tiene características que las pueden hacer parecer distintas o similares a otras, estas son las características tipológicas como son: la composición (nuclear, extensa o compuesta), el desarrollo (tradicional o moderna), la demografía (urbana, suburbana o rural), la ocupación (campesino, empleado o profesionista), la integración (integrada, o desintegrada), que son indispensables conocer ya que según Satir son útiles para determinar el grado de funcionalidad familiar.

Para Zavala et al. (2009), es un verdadero agente activo de una evolución social cambiante, pues una familia constituye jerarquía, orden, función y roles de cada uno de sus miembros, cuya organización está estrechamente relacionada con el entorno social, la familia se convierte así en 
subgrupos que comparten emociones y obligaciones constantes, lo que puede ocasionar afecciones en todo el sistema, que a su vez afectaría directa o indirectamente a sus miembros.

Rangel, Valerio, Patiño, \& García (2004), señalan que el funcionamiento en la familia puede ser responsable de la conservación de factores saludables o del padecimiento de una enfermedad entre los miembros, de ahí parte la premisa de que la familia es la que establece reglas y debe hacerlas cumplir, pues al hablar de una familia funcional o disfuncional, se refiere a la capacidad que tiene el sistema familiar para enfrentar y superar cada crisis por la que esté atravesando.

Una familia funcional se define por las cualidades que posee ésta para ser capaz de mantener armonía en su entorno, que permita de una manera u otra proveer el desarrollo individual de sus miembros, en concreto, son familias que generalmente no padecen trastornos psicológicos significativos y saben sobrellevar las crisis familiares; que si bien, no son difíciles de tolerar. Hay que tomar en cuenta que la funcionalidad familiar como tal se logra a partir de consolidar elementos dentro del sistema familiar como la comunicación, respeto, resolución de conflictos, flexibilidad, tolerancia de emociones y criterios variados, unión, manejo del estrés, etc., (González, Gimeno, Meléndezl, \& Córdoba, 2012).

Según Zavala et al., (2009), asevera que la familia está conformada por un sistema de miembros los cuales han decidido residir juntos o interrelacionarse afectivamente con el objetivo primario de apoyarse unos con otros. Lo que hace que los lazos entre los miembros sean más sólidos, dado que el apoyo mutuo puede contribuir a que dicha relación difícilmente se rompa.

Una familia que se sabe comunicar, favorece vínculos futuros, y permite intercambio de información dentro del sistema, la serenidad comunicativa permite altos grados de comprensión (Morales, 2010). Para este autor, los lazos afectivos pobres, producen sistemas de comunicación poco claros y poco directos, además de que los problemas de convivencia, la invasión en el espacio personal del otro, el rechazo, la falta de respeto, son indicadores de que puedan producirse desequilibrios en el núcleo familiar

Hernández, Cargill, \& Gutiérrez, (2012) indican que la salud en lo concerniente al completo bienestar familiar va a depender de la interrelación entre tres factores; los personales; que se tratan de aspectos biológicos, sociales y psicológicos, los factores propios de la unidad familiar; que corresponden a la estructura, la funcionalidad, economía, y el afrontamiento a las crisis y en tercer lugar están los factores sociológicos; que se refieren a aspectos sociales como de la comunidad y sociedad en general. 


\section{Según el DSM-V guía de consulta de los criterios diagnósticos (2013).Trastorno del espectro del autismo 299.00 (F84.0)}

En lo referente a las deficiencias persistentes en la comunicación social y en la interacción social en diversos contextos, manifestado por lo siguiente, actualmente o por los antecedentes (los ejemplos son ilustrativos pero no exhaustivos):

1. Las deficiencias en la reciprocidad socioemocional, varían, por ejemplo, desde un acercamiento social anormal y fracaso de la conversación normal en ambos sentidos pasando por la disminución en intereses, emociones o afectos compartidos hasta el fracaso en iniciar o responder a interacciones sociales.

2. Las deficiencias en las conductas comunicativas no verbales utilizadas en la interacción social, varían, por ejemplo, desde una comunicación verbal y no verbal poco integrada pasando por anomalías del contacto visual y del lenguaje corporal o deficiencias de la comprensión y el uso de gestos, hasta una falta total de expresión facial y de comunicación no verbal.

3. Las deficiencias en el desarrollo, mantenimiento y comprensión de las relaciones, varían, por ejemplo, desde dificultades para ajustar el comportamiento en diversos contextos sociales pasando por dificultades para compartir juegos imaginativos o para hacer amigos, hasta la ausencia de interés por otras personas.

Especificar la gravedad actual: La gravedad se basa en deterioros de la comunicación social y en patrones de comportamiento restringido y repetitivo

B. Patrones restrictivos y repetitivos de comportamiento, intereses o actividades, que se manifiestan en dos o más de los siguientes puntos, actualmente o por los antecedentes (los ejemplos son ilustrativos pero no exhaustivos):

1. Movimientos, utilización de objetos o habla estereotipados o repetitivos (p. ej., estereotipias motoras simples, alineación de los juguetes o cambio de lugar de los objetos, ecolalia, frases idiosincrásicas).

2. Insistencia en la monotonía, excesiva inflexibilidad de rutinas o patrones ritualizados de comportamiento verbal o no verbal (p. ej., gran angustia frente a cambios pequeños, dificultades con las transiciones, patrones de pensamiento rígidos, rituales de saludo, necesidad de tomar el mismo camino o de comer los mismos alimentos cada día). 
3. Intereses muy restringidos y fijos que son anormales en cuanto a su intensidad o foco de interés (por ejemplo., fuerte apego o preocupación por objetos inusuales, intereses excesivamente circunscritos o perseverantes).

4. Hiper- o hiperactividad a los estímulos sensoriales o interés inhabitual por aspectos sensoriales del entorno (p. ej., indiferencia aparente al dolor/temperatura, respuesta adversa a sonidos o texturas específicos, olfateo o palpación excesiva de objetos, fascinación visual por las luces o el movimiento).

Tanto el autismo como el trastorno del espectro autista comparten diferentes nombres con un el mismo significado "Ambos se refieren a los casos que tienen en común la clásica "Tríada de Wing": caracterizada por problemas en la comunicación, déficits en las habilidades sociales y por un repertorio de intereses restringido" (Dos Santos, Becker, Ranzan, Winckler, \& Ohlweiler, 2013) con lo que concuerda (Guzmán, Putrino, Martínez, \& Quiroz, 2017), añadiendo que dicha triada está constituida por "déficits en relaciones sociales, en comunicación, y en flexibilidad de intereses e imaginación".

\section{Familias de niños con autismo}

Las familias de niños con autismo no son distintas al resto de familias. Como expresa King, et al., (2006), en la que afirma, cada familia es diferente y única, exigen comprender los sistemas de creencia de las familias y sus procesos de adaptación. Es decir, se ajustan a los cambios con la ayuda de sus miembros al igual que las demás familias, con sus excepciones, es ahí donde la funcionalidad familiar entra en juego, pues en el caso de las familias de niños autistas, desarrollan modos y habilidades para compreder el mundo de su hijo y adaptarlo al mismo, en el que las prioridades son importantes y el aporte familiar de cada miembro es fundamental al igual que el de la comunidad. También destaca la capacidad y resistencia de las familias y la manera en la que logran modificar su mundo y como crean estrategias para adaptarse a los cambios por y para su hijo, la familia y el mundo.

\section{Material y métodos}

Esta investigación, se desarrolló bajo un enfoque cualitativo, mediante la cual se describen las variables, a través del método deductivo inductivo se intenta establecer una relación entre ambas variables, por lo que se apoya en el método bibliográfico y estadístico. En el espacio práctico se aplicó el instrumento del APGAR familiar y se elaboró un cuestionario que evaluó la interacción social de niños y niñas con el Trastorno del Espectro Autista. 
Durante el proceso de recogida de datos, se realizó una explicación previa a los participantes sobre los detalles de la investigación a realizar y lo que se pretendía lograr con el estudio. Para llevar a cabo esta investigación se contó con la participación de 18 niños y niñas pertenecientes a la Fundación "Un lugar para tus sueños" de la Ciudad de Portoviejo, con sus respectivas familias, por lo que se procedió a seleccionar a niños y niñas con el diagnóstico de Autismo, posterior a ello, se realizaron las instrucciones sobre cada test. Por su parte, el APGAR familiar, al estar diseñado para todos los miembros de la familia, fue resuelto por los padres y únicamente por los niños y niñas que cuyas características y condiciones individuales propias del trastorno permitiera que lo resolviera.

Se diseñó un cuestionario para evaluar la interacción social fue elaborado con el fin de valorar la percepción de uno de los progenitores del niño/a, por lo que debía ser respondido por quien lo conociera mejor, para garantizar que la respuesta se acercara a su realidad.

Se empleó el instrumento APGAR familiar, que muestra cómo perciben los miembros de la familia el nivel de funcionamiento de la unidad familiar de forma global, incluyendo a los niños ya que es aplicable a la población infantil (Suarez \& Alcalá, 2014). Además, permite asignar una valoración a la percepción de los miembros de una familia sobre su nivel de funcionamiento familiar de manera general, cuyo resultado se ve reflejado por el valor numérico que los miembros puntúan de acuerdo a la percepción individual que estos tienen en un momento determinado, la información que se obtiene es de gran ayuda para el investigador, puesto que basta con sumar los cinco parámetros que se evalúan, cuya puntuación oscila entre 0 y 20, en el caso de los adultos y 0 a 10 en los niños, para indicar una baja, mediana o alta satisfacción del funcionamiento de la familia, tabla 1.

Se creó un cuestionario que consta de 14 afirmaciones que permitiría evaluar la interacción social en niños con el Trastorno del Espectro Autista, bajo la percepción de sus padres, por lo que se procedió al diseño del mismo, estableciendo categorías que se relacionan con la interacción social en niños y niñas con TEA, teniendo como alternativas de respuesta las opciones de Buena, Regular y Mala.

Las categorías consideradas son, las emociones, los niños con Autismo tienden a no imitar las acciones, gestos o vocalizaciones que otros hacen de manera espontánea (Fundación ASEMCO, 2000). Por ello esta categoría tiene como propósito conocer como el niño expresa sus emociones, identifican las suyas y la de los demás, y en qué medida reconoce los gestos propios y el de los demás. 


\begin{tabular}{|l|l|}
\hline $\begin{array}{l}\text { Puntuación Cuestionario del APGAR } \\
\text { Familiar }\end{array}$ & Funcionalidad \\
\hline $20-17$ puntos & Función Familiar Normal \\
\hline $16-13$ puntos & Disfunción Leve \\
\hline $12-10$ puntos & Disfunción Moderada \\
\hline menor o igual a 9 & Disfunción Severa \\
\hline
\end{tabular}

Tabla 1. Clasificación de las puntuaciones del Apgar Familiar.

La Fundación ASEMCO, (2000), hace alusión a los en esta categoría, se pretende identificar en qué medida el niño comparte sus pertenencias, como se califica su saludo, porque los niños con este trastorno no presentan un contacto visual bueno y/o no responde a la cara o a la sonrisa de otros, el respeto y la manera de referirse a los demás ya que no desarrollan lenguaje verbal, "o bien lo utiliza de manera poco convencional repitiendo literalmente lo que oye o ha oído (frases de otros, fragmentos de películas o canciones)..

El tipo de juego, incluye parámetros para evaluar la iniciativa, el juego solitario y la integración grupal. Ya que suelen no mostrar interés en los otros niños de su edad o no saben iniciar juegos con ellos (Fundación ASEMCO, 2000). El juego interactivo, tiene como objetivo conocer como es la interacción con otros niños y en qué medida sigue las reglas de los juegos. Aunque generalmente los niños con este espectro tienden a aislarse, o "parece estar más interesado en los objetos que en las personas.

Las situaciones sociales, hacen alusión a como el niño expresa lo que quiere y siente en reuniones (familiares, laborales, sociales), ya que tienden a hacer berrinches exagerados y a veces sin tener un motivo aparente (Fundación ASEMCO, 2000). Además esta categoría describe la sociabilidad con sus pares y la manera en la que los padres califican como procede públicamente cuando no le gusta o no quiere algo.

Todos estos conceptos categóricos se orientan a obtener información sobre la interacción del niño/a con TEA, por lo que es necesaria la observación y completa sinceridad de sus padres al resolverlo.

Oportunamente éste cuestionario fue validado por especialistas en psicología clínica y psicopedagogos.

Para la realización de esta investigación se contactó en primera instancia con la "Fundación un lugar para tus sueños” de la ciudad de Portoviejo-Manabí, Ecuador, en la que se realizó una selección únicamente de los/las niños/as con TEA, acto seguido se realizó un contacto directo con 
sus respectivas familias, en la que 18 de ellas accedieron a ser partícipes de este estudio, a partir de lo cual consintieron la toma de los cuestionarios antes mencionados, mismos que fueron analizados a detalle por las autoras del presente artículo.

\section{Resultados}

Los resultados reflejan que el cuestionario APGAR familiar, se observan mejores resultados en lo referente a la función familiar normal, se aprecian en la tabla 2.

\begin{tabular}{|l|l|l|}
\hline $\begin{array}{l}\text { No. Familia } \\
\text { Código }\end{array}$ & $\begin{array}{l}\text { Puntuación Cuestionario } \\
\text { APGAR Familiar }\end{array}$ & Funcionalidad \\
\hline 001 & 20 & Función Familiar Normal \\
\hline 002 & 20 & Función Familiar Normal \\
\hline 003 & 19,5 & Función Familiar Normal \\
\hline 004 & 19,5 & Función Familiar Normal \\
\hline 005 & 19 & Función Familiar Normal \\
\hline 006 & 18 & Función Familiar Normal \\
\hline 007 & 18 & Función Familiar Normal \\
\hline 008 & 17 & Función Familiar Normal \\
\hline 009 & 17 & Función Familiar Normal \\
\hline 0010 & 16,7 & Función Familiar Normal \\
\hline 0011 & 16 & Disfunción Leve \\
\hline 0012 & 16 & Disfunción Leve \\
\hline 0013 & 15 & Disfunción Leve \\
\hline 0014 & 15 & Disfunción Leve \\
\hline 0015 & 12 & Disfunción Moderada \\
\hline 0016 & 12 & Disfunción Moderada \\
\hline 0017 & 12 & Disfunción Moderada \\
\hline 0018 & 11,5 & Disfunción Moderada \\
\hline
\end{tabular}

Tabla 2. Resultados obtenidos del Cuestionario del APGAR familiar

En cuanto a la pregunta para evaluar interacción social en niños y niñas con TEA, se observa que los resultados revelan que es regular.

\begin{tabular}{|l|l|}
\hline $\begin{array}{l}\text { No. Familia } \\
\text { Código }\end{array}$ & $\begin{array}{l}\text { Cuestionario para evaluar } \\
\text { Interacción Social en niños con TEA }\end{array}$ \\
\hline
\end{tabular}




\begin{tabular}{|l|l|}
\hline 001 & Buena \\
\hline 002 & Buena \\
\hline 003 & Buena \\
\hline 004 & Buena \\
\hline 005 & Buena \\
\hline 006 & Buena - Regular \\
\hline 007 & Regular \\
\hline 008 & Regular \\
\hline 009 & Regular \\
\hline 0010 & Buena \\
\hline 0011 & Mala \\
\hline 0012 & Regular \\
\hline 0013 & Mala \\
\hline 0014 & Mala \\
\hline 0015 & Regular \\
\hline 0016 & Regular \\
\hline 0017 & Regular \\
\hline 0018 & Regular \\
\hline
\end{tabular}

Tabla 3. Resultados del Cuestionario aplicado para evaluar interacción social en niños y niñas con TEA

En lo concerniente a relación entre ambas variables, para evaluar interacción social en niños con TEA, se aprecia que es regular, lo relacionado a la parte comunicativa, y por lo general ausencia de sonrisas y de expresiones verbales y no verbales, tabla 4.

\section{Discusión}

Cuando una familia que es más funcional que otra, se debe a la obediencia de las normas y funciones que cada uno tenga a su cargo, y a su vez que tengan los recursos necesarios para adaptarse fácilmente a los cambios que ésta requiera (Valladares, 2008). En esta investigación se refleja que 10 de las 18 familias participantes con niños y niñas con el trastorno del espectro autista tienen una función familiar normal, mismas de las cuales 6 de los hijos de estas familias tienen una interacción social buena, lo que indica que la funcionalidad familiar en estos casos fomentan una buena interacción social en los hijos de estas familias, pues adaptarse a las necesidades del niño involucra a todos los miembros de la familia, la calidad de sus relaciones y del apoyo externo, por lo que se concuerda con otros autores (Martínez \& Bilbao, 2008). 


\begin{tabular}{|c|c|c|c|}
\hline $\begin{array}{l}\text { No. } \\
\text { Familia } \\
\text { Código }\end{array}$ & Funcionalidad & $\begin{array}{l}\text { Puntuación } \\
\text { Cuestionario APGAR } \\
\text { Familiar }\end{array}$ & $\begin{array}{l}\text { Cuestionario para } \\
\text { evaluar Interacción } \\
\text { Social en niños con TEA }\end{array}$ \\
\hline 001 & Función Familiar Normal & 20 & Buena \\
\hline 002 & Función Familiar Normal & 20 & Buena \\
\hline 003 & Función Familiar Normal & 19,5 & Buena \\
\hline 004 & Función Familiar Normal & 19,5 & Buena \\
\hline 005 & Función Familiar Normal & 19 & Buena \\
\hline 006 & Función Familiar Normal & 18 & Buena - Regular \\
\hline 007 & Función Familiar Normal & 18 & Regular \\
\hline 008 & Función Familiar Normal & 17 & Regular \\
\hline 009 & Función Familiar Normal & 17 & Regular \\
\hline 0010 & Función Familiar Normal & 16,7 & Buena \\
\hline 0011 & Disfunción Leve & 16 & Mala \\
\hline 0012 & Disfunción Leve & 16 & Regular \\
\hline 0013 & Disfunción Leve & 15 & Mala \\
\hline 0014 & Disfunción Leve & 15 & Mala \\
\hline 0015 & Disfunción Moderada & 12 & Regular \\
\hline 0016 & Disfunción Moderada & 12 & Regular \\
\hline 0017 & Disfunción Moderada & 12 & Regular \\
\hline 0018 & Disfunción Moderada & 11,5 & Regular \\
\hline
\end{tabular}

Tabla 4. Relación entre ambas variables.

Por otra parte, 1 de estas 10 familias con funcionalidad normal posee un hijo con una interacción buena-regular, lo que significa que la relación familiar fomenta positivamente una buena interacción, puesto que "la alteración de cualquier miembro de una familia afecta al conjunto, la realidad de un niño con TEA trastoca todos los niveles de responsabilidad, madurez, dedicación y recursos de los que dispone la familia" (Martínez \& Bilbao, 2008). Y 3 de las 10 familias con función familiar normal, sus hijos poseen una interacción social regular, lo que muestra que a mayores niveles de funcionalidad familiar, la interacción social de sus hijos es más alta. Mismo que asevera que la participación de los miembros de las familias es de gran importancia y demuestra tener efectos positivos en niños con TEA, pues la familia está preparada para lidiar con las adversidades, sobre todo en el espacio social en el que este se desenvuelve (García, 2016). 
De las 18 familias participantes 4 tienen disfunción leve, de las cuales 3 de sus hijos tienen una interacción social mala, pues debido la disfuncionalidad familiar no se brindan los recursos necesarios para que haya una comunicación positiva entre el niño y el exterior, Martínez \& Bilbao (2008), indican que es necesario conocer a profundidad las características de dichos trastornos y aceptar las importantes limitaciones que implican a nivel de las relaciones sociales, la comunicación y la imaginación. Lo que se atribuye al desgaste y frustración de los padres ante su proceso de afrontar el diagnóstico de su hijo. La vida familiar se complica ante el diagnóstico del autismo, y afrontarlo lleva tiempo. Por otra parte 1 de esas 4 familias tienen un hijo cuya interacción social es regular, pues a pesar de la disfuncionalidad en la que vive esta familia, ha encontrado los mecanismos necesarios para preparar a su hijo hacia una buena interacción social. Se concuerda con otras investigaciones, que hacen alusión a estudios similares sobre este tema Ayuda, Llorente, Martos, Rodríguez, \& Olmo, (2012), en su investigación sobre programas de formación para padres de niños con autismo, plantean que los padres presentan dificultades derivadas de tener un niño con TEA, e indicaban que las familias cumplen un papel muy importante en la vida del niño que se debería considerar, pues hay beneficios directos que se generan sobre los niños con TEA tras la participación de sus padres, ésto debido a que funcionalmente la familia necesita de sus miembros para funcionar sólidamente.

Por otra parte 4 de las 18 familias participantes tienen una disfunción moderada, y pese a ello sus hijos poseen una interacción social regular, ésto debido a que en algunos casos depende de las características propias de cada niño y de su familia, este resultado se puede relacionar con una investigación sobre fomentar habilidades sociales y comunicativas a través del arte en niños con TEA, por lo que se concuerda con Coy \& Martín, (2017), no carecen de capacidades, sino que funcionan de una manera que no es la habitual; por lo tanto, con los medios y apoyos adecuados, pueden desarrollar muchas de las funciones que desempeña cualquier persona. Es decir que su resultado se reflejó en que los participantes disminuyeron su agresividad y ansiedad con sus familiares por desarrollar nuevas destrezas. 


\section{Conclusiones}

Se concluye que los niveles de Funcionalidad Familiar y la Interacción social de los niños y niñas con el Trastorno del Espectro Autista (TEA) pertenecientes a la "Fundación un lugar para tus sueños" de la ciudad de Portoviejo - Manabí - Ecuador, está íntimamente relacionado, pues un nivel de funcionalidad familiar normal contribuye significativamente a que la interacción social de los/las niños/as con TEA se adapte al medio en el que se desarrollan, por el contrario, si el nivel de funcionalidad familiar es leve o moderado puede dificultar la interacción social en los y las niñas con TEA, debido a que los padres pueden indirectamente ocasionar dificultad en las habilidades sociales y comunicativas del/la niño/a, ésto con ciertas excepciones, dado que los datos obtenidos en esta investigación revelan que la mayor parte de los participantes muestran un nivel de Interacción Social Regular, y en algunos casos, no depende necesariamente del nivel de Funcionalidad Familiar, sino más bien de la gravedad y de las características propias del trastorno, dado que el trastorno estudiado por tratarse de un espectro como tal, varía dependiendo del caso, grado de autismo, y peculiaridades de los participantes.

\section{Bibliografía}

Asociación Americana de Psiquiatría. (2013). Guía de consulta de los criterios diagnósticos del DSM 5. Arlington, VA.

Ayuda, R., Llorente, M., Martos, J., Rodríguez, L., \& Olmo, L. (2012). Medidas de estrés e impacto familiar en padres de niños con trastornos del espectro autista antes y después de su participación en un programa de formación. Revista de neurología, 54(1), 6. Recuperado de: https://philarchive.org/archive/LACLIDv2

Baña, M. (2015). El rol de la familia en la calidad de vida y la autodeterminación de las personas con trastorno del espectro del autismo. Ciencias Psicológicas, 9(2), 323-336.

Coy, L., \& Martín Padilla, E. (2017). Habilidades sociales y comunicativas a través del arte en jóvenes con trastorno del espectro autista (TEA). Estudios pedagógicos (Valdivia), 43(2), 262. Recuperado de https://scielo.conicyt.cl/scielo.php?pid=S0718-07052017000200003 $\underline{\text { \&script }=\text { sci_arttext\&tlng=en }}$

Dos Santos, R., Becker, M., Ranzan, J., Winckler, M.., \& Ohlweiler, L. (2013). Evolución de los niños con retraso del desarrollo y conductas del espectro autista. Medicina (Buenos Aires), 73 
(1), 16-19. Recuperado de http://www.medicinabuenosaires.com/revistas/vol73-13/supl-1/1619-Supl\%203-A\%20-\%20OK.pdf

Fundación ASEMCO. (2000). Trastorno del Espectro Autista. Recuperado de Fundación ASEMCO, recuperado de: https://www.asemco.org/trastornos-espectro-autista-tea.php

García, A. (2016). La participación de las familias en la atención temprana de los niños con trastorno del espectro autista, 140. Recuperado de: https://gredos.usal.es/ jspui/handle/10366/137006

González, F., Gimeno, A., Meléndezl, J., \& Córdoba, A. (2012). La percepción de la funcionalidad familiar: Confirmación de su estructura bifactorial. Escritos de Psicología (Internet), 5(1), 34-39. Recuperado de http://scielo.isciii.es/scielo.php?script=sci_arttext\&pid=S1989_ $\underline{38092012000100005}$

Guzmán, G., Putrino, N., Martínez, F., \& Quiroz, N. (2017). Nuevas tecnologías: Puentes de comunicación en el trastorno del espectro autista (TEA). Terapia psicológica, 35(3), 3. Recuprado de: https://scielo.conicyt.cl/pdf/terpsicol/v35n3/0716-6184-terpsicol-35-030247.pdf

Hernández, L., Cargill, N. , \& Gutiérrez, G. (2012). Funcionalidad familiar y conducta de riesgo en estudiantes denivel medio superior Jonuta, Tabasco 2011. Salud en Tabasco, 18(1), 15. Recuperado de: https://www.redalyc.org/articulo.oa?id=487/48724427004

King, G., Zwaigenbaum, L., King, S., Baxter, D., Rosenbaum, P., \& Bates, A. (2006). Cambios en los sistemas de convicciones/valores de las familias de niños con autismo y síndrome de Down. Revista Síndrome de Down, 23, 55-57.

Martínez, M., \& Bilbao, M. (2008). Acercamiento a la realidad de las familias de personas con autismo. Intervención Psicosocial, 17(2), 220-223. Recuperado de: http://scielo.isciii.es/pdf/inter/v17n2/v17n2a09.pdf

Mendoza, L., Soler, E., Sainz, L., Gil, I., Mendoza, H., \& Pérez, C. (2006). Análisis de la dinámica y funcionalidad familiar en atención primaria. Archivos en medicina familiar, 8(1), 28. Recuperado de: https://www.medigraphic.com/pdfs/medfam/amf-2006/amf061d. pdf 
Miguel, A. (2006). El mundo de las emociones en los autistas. Teoría de la Educación. Educación y Cultura en la Sociedad de la Información,, 7(2), 169-182.

Montagut, M., D'Ocon, A., \& Pastor , G. (2017). Interacción social y comunicación temprana enhermanos de niños con diagnóstico de trastorno del espectro autista. Revista Infad de Psicología, 4(1), 373-387.

Morales, L. B. (2010). Autismo, familia y calidad de vida. Cultura: Revista de la Asociación de Docentes de la USMP, 24(8), 1-20.

Oviedo, N., Manuel, L., de la Chesnaye, E., \& Guerra, C. (2015). Aspectos genéticos y neuroendocrinos en el trastorno del espectro autista. Revista Elsevier, pag, 6-10. Recuperado de: https://reader.elsevier.com/reader/sd/pii/S1665114615000349?token=3ABE7E1EB02259A CFE81D73B788DC8CB6EFFD1E66B82C305A9D6B9FA3B9B482051D3B0600E306436871

\section{DC91D8A50E}

Rangel, J., Valerio, L., Patiño, J., \& García, M. (2004). Funcionalidad familiar en la adolescente embarazada. Revista de la Facultad de Medicina, 47(1), 24.

Suarez, M., \& Alcalá Espinoza, M. (2014). Apgar familiar: una herramienta para detectar disfunción familiar. Revista Médica La Paz, 20(1), 53-56. Recuperado de: http://www.scielo.org.bo/scielo.php?script=sci_arttext\&pid=S1726-89582014000100010

Valladares, A. (2008). La familia. Una mirada desde la Psicología. MediSur, 6(1), 2-7.

Zavala, M. , Ríos, M., García, G., \& Rodríguez, C. (2009). Funcionalidad familiar y ansiedad enpacientes adultos con enfermedad crónica. Aquichan, 9(3), 259-270. Recuperado de: https://aquichan.unisabana.edu.co/index.php/aquichan/article/view/1524/1830 